\title{
Capacity Analysis Based on Using Adaptive Antenna Arrays Jointly Optimized for Trunking and Spatial Efficiency
}

\author{
Ivan Howitt \\ Wireless and Signal Processing Laboratory, Electrical Engineering and Computer Science Department, \\ University of Wisconsin Milwaukee, P.O. Box 784, Milwaukee, WI 53201, USA \\ Email:howitt@uwm.edu \\ Fahed H. Awad \\ Wireless and Signal Processing Laboratory, Electrical Engineering and Computer Science Department, \\ University of Wisconsin Milwaukee, P.O. Box 784, Milwaukee, WI 53201, USA \\ Email:awad@uwm.edu
}

Received 24 July 2001 and in revised form 28 February 2002

\begin{abstract}
Cellular systems using adaptive antennas for spatial processing have been shown to provide an increase in capacity. When employing adaptive antennas, the standard approach to achieve capacity gain has been to maximize the link quality between the mobile and base station via optimum combining. This paper presents an alternative optimization approach based on jointly optimizing the adaptive antenna array to maximize the spatial and trunking efficiencies. An upper bound on the joint optimization was formulated to evaluate the capacity. Based on this upper bound, the effect of using adaptive antenna arrays to maximize the trunking efficiency was subsequently an outstanding issue. Monte Carlo simulations, substantiated with theoretical analysis, were used to evaluate this issue. Based on the analysis, utilizing the adaptive antennas to optimize trunking efficiency could increase the capacity by 2 to 4 times.
\end{abstract}

Keywords and phrases: adaptive antennas arrays, cellular capacity, trunking efficiency, spatial efficiency.

\section{INTRODUCTION}

The information age has accelerated the need for communications and the mobile society has brought about an everincreasing demand for access to untethered communications. Current wireless telephony services are dominated by the design constraints imposed by speech; whereas, the third generation systems are exploring concepts to handle mixed signal environments. The increase in demand and the ability to handle multimedia applications have increased the need to achieve greater spectral efficiency. To address these needs, spatial processing is being vigorously pursued in both academia and industry. Adaptive antennas are regarded by many within the industry as one component in meeting the service needs in future generation mobile networks $[1,2]$. At issue is the method for utilizing adaptive antennas that will achieve the greatest performance benefit.

Spectral efficiency, $\eta_{S}$, is a primary measure of effectiveness for wireless services, which can be expressed as [3]

$$
\eta_{S}=\eta_{B} \times \eta_{C} \times \eta_{T} \quad \text { erlang } / \mathrm{m}^{2} / \mathrm{Hz},
$$

where $\eta_{B}$ is the bandwidth efficiency. Spatial efficiency, $\eta_{C}$, provides a measure of the service's efficiency of providing traffic channels within a given area. Trunking efficiency, $\eta_{T}$, as used in this paper, is a measure of the offered traffic per channel in the network. Spatial filtering for interference reduction (SFIR) and space division multiple access (SDMA) are current methods for utilizing adaptive antennas. Both methods affect $\eta_{C}$ to achieve an improvement in $\eta_{S}$. SFIR and SDMA exploit spatial diversity between the signal of interest (SOI) and cochannel interfers by restricting signal reception to the directions that enhance the SOI's quality. For frequency division multiple access (FDMA) and/or time division multiple access (TDMA) systems, the method in which $\eta_{C}$ is influenced is distinct for each spatial processing technique. In FDMA/TDMA systems, frequency planning and cochannel reuse distances are central to network planning. SFIR is used to reduce the impact of cochannel interference from typically the first tier of cochannel cells, thereby allowing a reduction in the reuse distance for the same quality of service (QoS). On the other hand, SDMA allows multiple users to be assigned to the same traffic channel within 


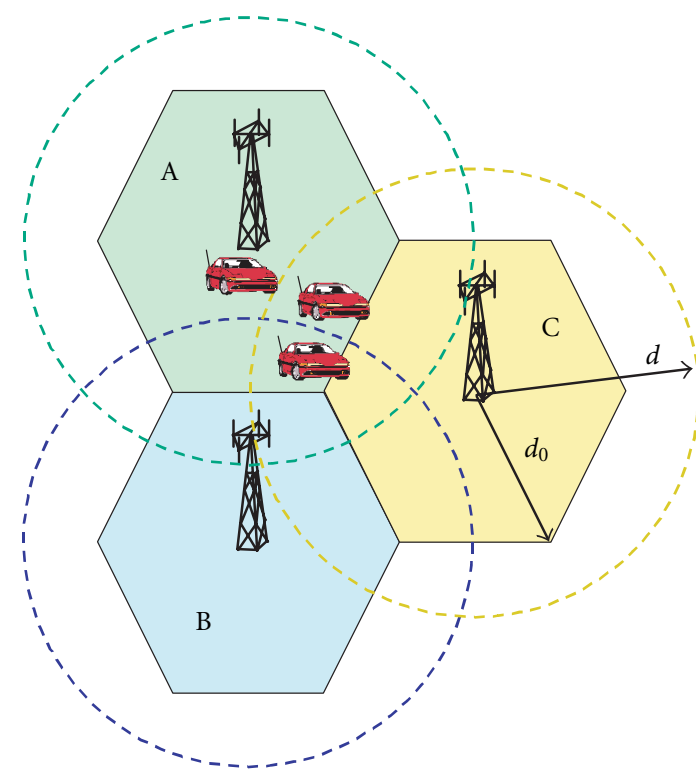

Figure 1: Example illustrating the advantage of using an adaptive antenna array to maximize the trunking efficiency.

a cell. Spatial diversity is again used to achieve the desired signal quality. The signal processing required is essentially the same for both methods, but the impact on $\eta_{C}$ can be significantly different, depending on the underlying assumptions used in the analysis. For code division multiple access (CDMA) systems, network frequency planning is less restrictive, but cochannel interference limits the capacity and therefore, spatial filtering can affect CDMA performance.

An alternative approach for utilizing smart antenna arrays has been implemented commercially for CDMA/AMPS networks as reported in [4]. The method is based on adjusting each base station's (BS) antenna pattern in order to balance the traffic loads within each cell of the network. The antenna pattern is adjusted using phased array antennas at the BSs in the network, which are remotely configured by operators monitoring the traffic. Traffic channels available within the network are thereby more likely to be utilized and all cells in the network are less likely to exceed $100 \%$ loading. Therefore, the phased array is adapted to improve $\eta_{T}$ and thereby improve $\eta_{S}$. In this paper, the impact on $\eta_{S}$ is investigated when adaptive antennas at the BSs in a cellular network are adapted dynamically to maximize $\eta_{T}$. In Sections 3 and 4 , the simulation model and corresponding results are presented. In order to obtain a point of validation for the simulation model, an analytical solution was obtained based on a 1D network topology, as derived in Appendix A. A comparison of the analytical and simulation results is summarized in Section 3.2.

Figure 1 illustrates the concept for a three-cell network where each BS is equipped with an adaptive array. The hexagonal grid is used to illustrate the coverage region based on an omnidirectional antenna at each BS. Radius $d_{0}$ is the maximum radius to achieve the desired received signal-to-noise ratio (SNR). Radius $d$ is the corresponding maximum radius when an adaptive array is employed. If the three mobile stations (MSs) request service at the same time, then BS-A needs to have three traffic channels available, assuming omnidirectional antennas, in order to avoid blocking a service request. Even if SDMA is employed, three FDMA, TDMA, and/or CDMA traffic channels are still likely to be required for the scenario depicted in the figure. This is due to the spatial correlation between the received signals being too large to allow the same traffic channel to service all three users. Adapting the antenna array to maximize $\eta_{T}$, allows one traffic channel from each BS to be used to handle the offered traffic. Again, three orthogonal traffic channels are required, but with a $67 \%$ improvement in trunking efficiency at BS-A. Therefore, greater flexibility is provided to handle additional traffic.

A number of studies, both analytical and field, show that adaptive antennas can improve spectral efficiency. At issue is how the adaptive array can be used most effectively. As indicated by the simple example in the previous paragraph, maximizing $\eta_{C}$ may not always provide the best $\eta_{S}$. Likewise, the converse is also true, maximizing $\eta_{T}$ does not ensure the best $\eta_{s}$. Therefore, adapting the antenna array for the joint maximization of $\eta_{T} \times \eta_{C}$ seems appropriate. In Section 2, the concept of jointly optimizing the adaptive antenna array over $\eta_{T} \times \eta_{C}$ is developed further and an upper bound is formulated such that the capacity increase over an omnidirectional antenna can be evaluated. In order to evaluate the upper bound, the effect of using adaptive antenna arrays to maximize $\eta_{T}$ is required and is addressed in Sections 3 and 4 as indicated above.

\section{JOINT OPTIMIZATION CONCEPT}

A standard optimization strategy for adaptive antenna arrays to achieve spatial efficiency, $\max \left(\eta_{C}\right)$, is to mitigate the impact of channel impairments, by optimally combining the SOI's multipath components and by the suppression of cochannel interference. This is done independently for each MS assigned to the BS $[5,6,7,8,9,10]$. The adaptive antenna weights, $\mathbf{W}$, at the BS are adapted to maximize the QoS for the SOI, $S$,

$$
\mathbf{W}^{*}=\underset{\mathbf{W}}{\operatorname{argmax}}\left(\operatorname{SINR}_{S}\right),
$$

where SINR $_{S}$ is the signal-to-noise and interference ratio for signal $S$. Based on our research and the research of others $[11,12,13,14,15,16,17,18,19]$, using this approach in conjunction with SFIR can achieve a reuse factor reduction from 7 to either 3 or 1, with a corresponding capacity increase of either $133 \%$ or $600 \%$, respectively. The effectiveness of using an adaptive antenna for SDMA in an FDMA/TDMA system is somewhat less clear. From the results published in the literature $[13,14,15,20]$, a capacity increase over SFIR of approximately $40 \%-20 \%$ is indicated. The lower capacities were achieved with an antenna array of approximately 4 elements, while the higher capacities typically required in excess of 8-element antenna arrays. 
For CDMA-based systems, using an adaptive array can provide spatial diversity and reduce the impact of interference, especially under imperfect power control. Capacity and performance issues, when using adaptive antennas in CDMA based systems, have been addressed in $[21,22,23,24,25,26]$. Results in both $[24,25]$ indicate a $500 \%$ increase in capacity over an omnidirectional antenna using an antenna array of approximately 8 elements.

Using (2) to optimize the link quality between each BS to MS independently, appears to provide an increase in capacity regardless of the multiple access technique used by the cellular service. As indicated above, using (2) in conjunction with SFIR and/or SDMA maximizes $\eta_{c}$, but it does not take into account the impact that the adaptive antenna array may have on $\eta_{T}$. The following alternative approach, which optimizes the adaptive array to jointly maximize spatial efficiency and trunking efficiency, that is, $\max \left(\eta_{C} \times \eta_{T}\right)$, should achieve greater spectral efficiency. Letting $n$ represent the number of signals of interest within the coverage region of $b \mathrm{BSs}$, then the solution to $\max \left(\eta_{C} \times \eta_{T}\right)$ is based on adapting the weight matrix $\mathbf{W}=\left[\begin{array}{lll}\mathbf{W}_{1} & \cdots & \mathbf{W}_{b}\end{array}\right]$ where $\mathbf{W}_{j}, j=1, \ldots, b$, are the weight vectors corresponding to each BS in the network. The objective is to determine $\mathbf{W}^{*}$ such that

$$
\mathbf{W}^{*}=\underset{\mathbf{W},\left\{S_{i}\right\}}{\operatorname{argmax}}\left(\left|\left\{S_{i}\right\}\right| \mid \operatorname{Pr}\left(\operatorname{SINR}_{S_{i}} \leq \gamma_{i}\right) \leq \xi_{i}, i \in 1, \ldots, n\right),
$$

where $\left(\mid\left\{S_{i}\right\} \| \operatorname{Pr}\left(\operatorname{SINR}_{S_{i}} \leq \gamma_{i}\right) \leq \xi_{i}\right)$ is the cardinality of the set of signals $\left\{S_{i}\right\}$ with their required QoS satisfied. The QoS is based on the outage probability, $\operatorname{Pr}(\operatorname{SINR} \leq \gamma)$, with the protection ratio $\gamma$. Equation (3) optimizes the adaptive array to maximize the number of signals that meet or exceed the desired QoS, that is, minimizes the blocking probability. Therefore, the solution to (3) maximizes spatial efficiency and trunking efficiency, $\max \left(\eta_{C} \times \eta_{T}\right)$. The direct implementation of (3) can be highly complex, since $\mathbf{W}^{*}$ needs to be determined for each user in the network. As required by (3), this means determining $\mathbf{W}^{*}$ for one user is dependent on the $\mathbf{W}^{*} \mathrm{~s}$ for all the other users. The formulation does suggest a more tractable two-step approach: dynamically adapt the coverage region of the $\mathrm{BS}$ based on the spatial distribution of the MS and then employ SFIR and/or SDMA to enhance the QoS.

In order to evaluate the capacity increase provided by an adaptive antenna array optimized based on (3), an upper bound on $\max \left(\eta_{C} \times \eta_{T}\right)$ is formulated. A simple upper bound is given by

$$
\max \left(\eta_{C} \times \eta_{T}\right) \leq \max \left(\eta_{C}\right) \max \left(\eta_{T}\right) .
$$

The first term in the bound, $\max \left(\eta_{C}\right)$, is based on using (2) to optimize the adaptive antenna weights. The corresponding capacity gains have been addressed in the literature, as reviewed above. Equality in (4) is unlikely, since $\eta_{C}$ and $\eta_{T}$ are not independent functions. As is evident from (3), tradeoffs are required in determining the optimal set of weights. In (2), the weights are optimized to maximize the SINR of each MS to BS assignment, with assignments typically made to minimize cochannel interference. In (3), this constraint is relaxed by allowing MSs to be assigned to BSs that require an increase in directional signal power and hence cause an increase in cochannel interference. The trade-off involves balancing the degradation in network performance caused by the increase in cochannel interference with the benefit of improved load balancing. This required trade-off is not reflected in the bound presented on the right-hand side of (4).

In order to evaluate $\max \left(\eta_{T}\right)$, a simplified version of (3) is used,

$$
\begin{aligned}
\mathbf{W}^{*}=\underset{\mathbf{W},\left\{S_{i}\right\}}{\operatorname{argmax}}\left(\left|\left\{S_{i}\right\}\right| \mid D\left(B S_{j}, M S_{i}\right) \leq d,\right. \\
\\
\forall j \in 1, \ldots, b ; \forall i \in 1, \ldots, n),
\end{aligned}
$$

where $\left(\mid\left\{S_{i}\right\} \| D\left(B S_{j}, M S_{i}\right) \leq d\right)$ is the cardinality of the set of signals $\left\{S_{i}\right\}$ within the network assigned to a BS, and $D\left(B S_{j}, M S_{i}\right)$ is a distance measure between the $j$ th BS and the $i$ th MS. Based on (5), the adaptive array is adapted to provide range extension. The range extension is then used to optimize the BS to MS assignment in order to minimize the blocked traffic, that is, $\min \left(n_{b}\right)$, where $n_{b}$ is the number of users blocked in the network. Alternatively, (5) can be viewed as maximizing the number of channels utilized by the network. In (5), the QoS is assumed sufficient, given that the MS is within distance $d$ of the assigned BS. This imposes the requirement that cochannel interference is mitigated by either using orthogonal traffic channels, SFIR/SDMA, channel allocation to minimize interference, or some combination of the techniques to ensure that the required QoS is obtained. The solution for $\min \left(n_{b}\right)$ based on (5) always exists and, in general, the MS to BS assignment that achieves the $\min \left(n_{b}\right)$ is not unique. However, additional constraints could be imposed to obtain the solution that minimizes cochannel interference, power, and/or hand-off frequency.

The optimization of (3) or (5) will be dependent on the multiple access technique(s) used for the service. For example, the downlink for a CDMA service requires simultaneous transmission to all users assigned to the BS in order to maintain orthogonal coding. Therefore, for CDMA the solution to $W^{*}$ in (5) can be viewed as a reshaping of the cell boundaries based on the traffic load (similar to the approach used in [4]). The resulting trunking efficiency obtained for CDMA using this approach will most likely be different than optimizing $W^{*}$ for an individual MS, as may be possible in a FDMA/TDMA based service. A goal of the paper is to present a general upper bound. Tighter bounds could be obtained by utilizing system specific characteristics.

In evaluating $\max \left(\eta_{T}\right)$, there are two important issues:

(1) the feasible range for extending the BS coverage using an adaptive array, and

(2) the spatial traffic model used in the evaluation. Based on the simulation results presented in [27], using a circular adaptive antenna array at the BS could extend the coverage range by a factor of 2.8 or 5.5 with a 10- or 100-element antenna array, respectively. This conclusion is based on a $3^{\circ}$ scattering angle, path loss 
exponent of 4 and a single MS. An analytical study [28], based on the match filter bound on the bit error rate probability, indicates the expected coverage range for a 10-element uniform linear array (ULA) is nearly double that of an omnidirectional antenna. The expectation is over variation in the angle spread and the pointing angle between the BS and MS. Based on these results, if the adaptive array is used solely to provide range extension, then a range increase from 1.5 to 2.5 seems feasible. Using the adaptive antenna array to achieve directional gain as well as spatial processing could incur an increase in the number of antenna elements required to obtain the desired performance.

In [29], complex deployment scenarios are specified as test environments for third generation Universal Mobile Telecommunication System radio services, based on probabilistic models for user activity and user movement within the network coverage area. A mixture of packet-switched data services along with circuit-switched services at various data rates are considered. In third generation systems, one method for handling high data rate users is to use multiple channels to provide high capacity links [30, 31, 32]. Under these environments, spatially correlated traffic with a nonuniform spatial distribution is likely. In order to evaluate $\max \left(\eta_{T}\right)$ based on optimizing (5), the important aspect of the traffic deployment is the active users' spatial distribution. This distribution can be viewed as a random process (RP) governed by the probabilistic models of user activity, movement and service types. The traffic models used in the analysis are based on evaluating the RP at a given instant in time. Therefore, the traffic models are random variables (RV) that describe the location of active users within the network coverage region at an instant in time. Two traffic models were considered. The first traffic model was used to provide a baseline, with the users' traffic spatially independent and identically distributed (iid), with a uniform distribution over the network coverage region. The second traffic model was based on a stochastic model developed to describe cell load activity within a network [33]. Based on empirical data of cell load activity, statistical analysis was used to analyze the hypothesis that the logarithm of the cellular traffic can be described by a Gaussian distribution. The hypothesis was rejected for only a small percentage of cases. The standard deviation for the log-normal distribution, $\sigma_{\mathrm{Tr}}$, was determined based on the empirical data with most values ranging from 3 to $4 \mathrm{~dB}$ erlang. This empirical study was used in developing the second traffic model as discussed in Section 3.1.

\section{MODEL DEVELOPMENT}

In order to analyze the capacity enhancement based on using adaptive antennas to maximize the trunking efficiency, the conditional blocking rate probability density function (pdf), $p_{\mathrm{Br}}\left(n_{b} \mid d / d_{0}\right)$, was evaluated, where $n_{b}$ is the number of blocked users in the network and $d / d_{0}$ is the relative range extension provided by the adaptive antenna array over an

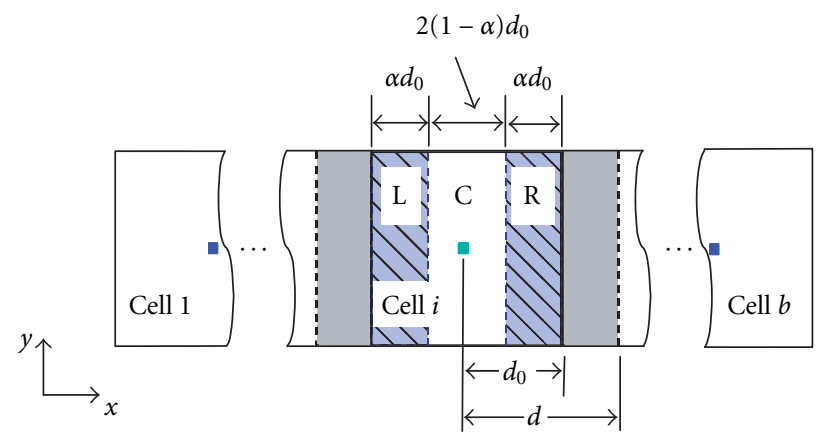

Figure 2: Linear grid cellular system used for 1D network model.

omnidirectional antenna. A closed form solution for the pdf is, in general, difficult to obtain due to the combinatorics involved with determining the number of blocked users when $d / d_{0}>1.0$. This difficulty can be observed in even the threecell network depicted in Figure 1. A Monte Carlo simulation was therefore used to estimate the pdf. In Section 3.1, the simulation model used in the analysis is presented.

Analytical results were tractable for a 1D network model, Figure 2. A derivation of the cumulative distribution function (cdf) for $d / d_{0}=1.0$ is presented in Appendix A.1. A corresponding upper bound and estimate of the cdf for $d / d_{0}>1.0$ are presented in Appendix A.2. The analytical results were used to show that the Monte Carlo results were consistent with analytical results for the 1D cellular system. A comparison of the $1 \mathrm{D}$ network model results is summarized in Section 3.2.

\subsection{Simulation model}

Monte Carlo simulations were used to estimate the conditional blocking rate pdf, $p_{\mathrm{Br}}\left(n_{b} \mid d / d_{0}\right)$. For the simulation results presented in Section 4, a 2D network model was used. The 2D network model consisted of a $\sqrt{b} \times \sqrt{b}$ hexagonal grid of cells with each of the $b$ BS located at the center of a hexagon. The BS coverage range was $d_{0}$, with an omnidirectional antenna, and $d$, with an adaptive antenna array. The network coverage region was defined as the area contained within the union of the circles with radius $d_{0}$ and the circles centered at each BS. Therefore, the region over which the users are distributed within the network remains the same, regardless of the extension in coverage range provided by the adaptive array.

The cells within the network could each support up to $m$ traffic channels. The total traffic in the network at a given instant in time is $n$, where $n=b m G_{c}$ and $G_{c}$ is the offered traffic per channel in the network. Therefore, $G_{c}$ is the network trunking efficiency for which the corresponding blocking rate pdf is to be estimated. The analysis focuses on scenarios where sufficient channels are available in the network to service the users' requests, that is, $0<G_{c} \leq 1$ and examines the network's ability to be configured to provide coverage for the $n$ users.

As indicated in Section 2, the user spatial distribution can impact the trunking efficiency. Because of this, two RVs were 
used to spatially distribute the MS traffic load: Traffic Model I and Traffic Model II. For Traffic Model I, the MSs were iid with a uniform distribution over the network coverage area. For Traffic Model II, the traffic load at each cell was iid with a log-normal distribution. The log-normal distribution was zero mean with standard deviation, $\sigma_{\mathrm{Tr}}$. The values of $\sigma_{\operatorname{Tr}}$ used in the simulation were based on typical values reported in [33]. Based on the load distribution obtained using the log-normal RV, the combined traffic load over all $b$ cell sites was normalized to equal $n$. The resulting traffic load within each cell was spatially iid, with a uniform distribution. The approach used for Traffic Model II was consistent with the stochastic traffic model developed in [33] as discussed in Section 2.

To estimate $p_{\mathrm{Br}}\left(n_{b} \mid d / d_{0}\right)$, multiple trials were performed using the same parameters, that is, the following parameters were fixed for each experiment: $d / d_{0}, m, G_{c}, n, b$, and the Traffic Model (I or II). For each trial in an experiment, the following steps were performed:

(1) distribute user traffic load within the network coverage region based on Traffic Model I or II;

(2) assign MS to BS to achieve $\min \left(n_{b}\right)$;

(3) determine number of users blocked, $n_{b}$, and update histogram;

(4) terminate experiment when the expected value of $\operatorname{Pr}_{\mathrm{Br}}\left(n_{b} \mid d / d_{0}\right)$, for all $n_{b}$ was within \pm 0.01 , with a $95 \%$ confidence.

Based on the trials performed for the experiment, $p_{\mathrm{Br}}\left(n_{b}\right)$ $d / d_{0}$ ) was then estimated.

The assignment strategy, step 2, was the essential component in maximizing $\eta_{T}$, by minimizing $n_{b}$. The adaptive antenna weights were implicitly optimized based on (5). A heuristic was used to approximate $\min \left(n_{b}\right)$ and the details of the heuristic are given in Appandix B.

\subsection{Simulation model validation based on 1D statistical analysis}

In order to corroborate the results from the simulation model discussed in the previous section, statistical analysis was performed on a $1 \mathrm{D}$ network topology, Figure 2. In Appendix A.1, the blocking rate cdf, $P_{\mathrm{Br}}\left(n_{b} \mid d / d_{0}=1.0\right)$, is derived based on an omnidirectional antenna at the BS. A comparison of the blocking rate pdfs obtained with the analytical, $\left(p_{\mathrm{Br}}\left(n_{b} \mid d / d_{0}=1.0\right)\right)_{\mathrm{Th}}$, and simulation, $\left(p_{\mathrm{Br}}\left(n_{b} \mid d / d_{0}=1.0\right)\right)_{\mathrm{Sim}}$, are given in Figure 3 (line graphs used for clarity; pdfs are sequence of impulses at 0 to $n-1)$. The results were based on a $1 \mathrm{D}$ network with $b=10, m=5$, $G_{c}=1.0$, and $n=50$ users. The users were iid and uniformly distributed within the cellular system coverage area. The results from the simulation were consistent with the confidence interval used for estimating the blocking rate probabilities. The pdf comparison in Figure 3 reflects typical error results when $d / d_{0}=1.0$.

An analytical solution for the probability of blocking in the $1 \mathrm{D}$ network when $d / d_{0}>1.0$ was not tractable. The difficulty in formulating a solution was in determining

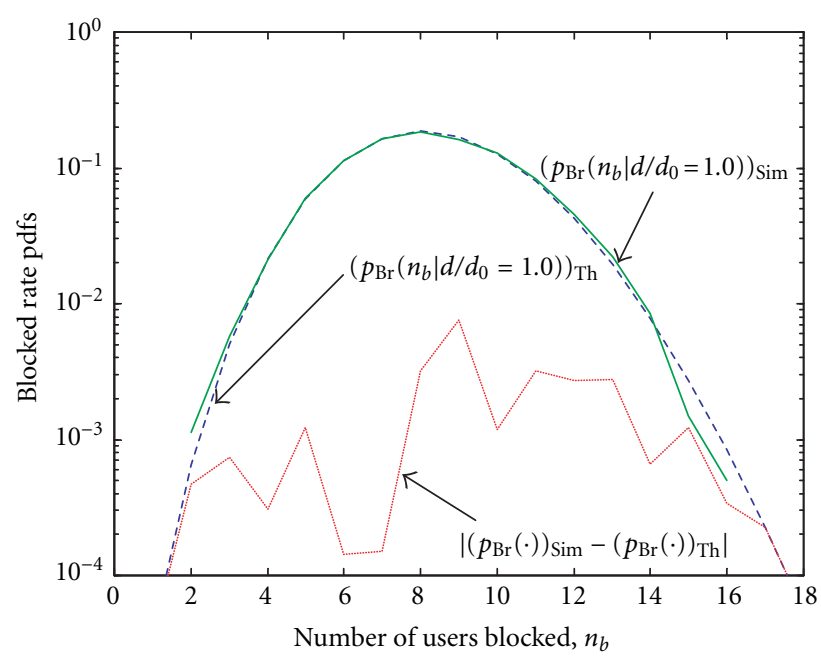

FIGURE 3: Comparison of the blocking rate pdf obtained based on analytical evaluation and simulation estimation. The pdf is for $1 \mathrm{D}$ model with $b=10, m=5, G_{c}=1.0, n=50$, and $d / d_{0}=1.0$.

the likelihood of the channels from adjacent cells being available to prevent blocking within their extended coverage region. An upper bound on the blocking rate cdf,

$$
\left(P_{\mathrm{Br}}\left(n_{b} \mid d / d_{0}=(1.0+\alpha)\right)\right)_{\text {Bound }} \geq P_{\mathrm{Br}}\left(n_{b} \mid d / d_{0}=(1.0+\alpha)\right)
$$

with $0 \leq \alpha \leq 1.0$, was formulated based on assuming that all $m$ channels from the adjacent cells were available within their extended coverage region. Thereby, a lower bound on the number of blocked users was obtained. The derivation for (6) is given in Appendix A.2. For small values of $G_{c}$ and $\alpha$, the bound should be tight, since the adjacent cells extended coverage region is small and the likelihood of an adjacent cell's channels being available to service the MSs within these regions is high. As either $G_{c}$ or $\alpha$ increases, the bound becomes less tight.

An estimate of the blocking rate cdf,

$$
\begin{aligned}
& \left(P_{\mathrm{Br}}\left(n_{b} \mid d / d_{0}=(1.0+\alpha)\right)\right)_{\mathrm{Est}} \\
& \quad \approx P_{\mathrm{Br}}\left(n_{b} \mid d / d_{0}=(1.0+\alpha)\right) \quad \text { with } 0 \leq \alpha \leq 1.0,
\end{aligned}
$$

was also formulated, Appendix A.2. The estimate is based on assuming that $\hat{M}$ channels from the adjacent cells were available in their extended coverage region, where $\hat{M}$ is the expected number of available channels based on the level of user activity within the network.

The analytical bound in (6) and analytical estimate in (7) were compared to the blocking rate probability estimates obtained by the simulation. In Figure 4, the blocking rate cdfs are compared based on a 1D network topology with $d / d_{0}=1.25, b=10, m=5, G_{c}=0.8$, and $n=40$. In Table 1 , the expected blocking rates, $\mu_{\mathrm{Br}}\left(n_{b} \mid d / d_{0}\right)$, obtained by the three approaches are provided, based on the $1 \mathrm{D}$ network and for several values of range extension and user activity. The 


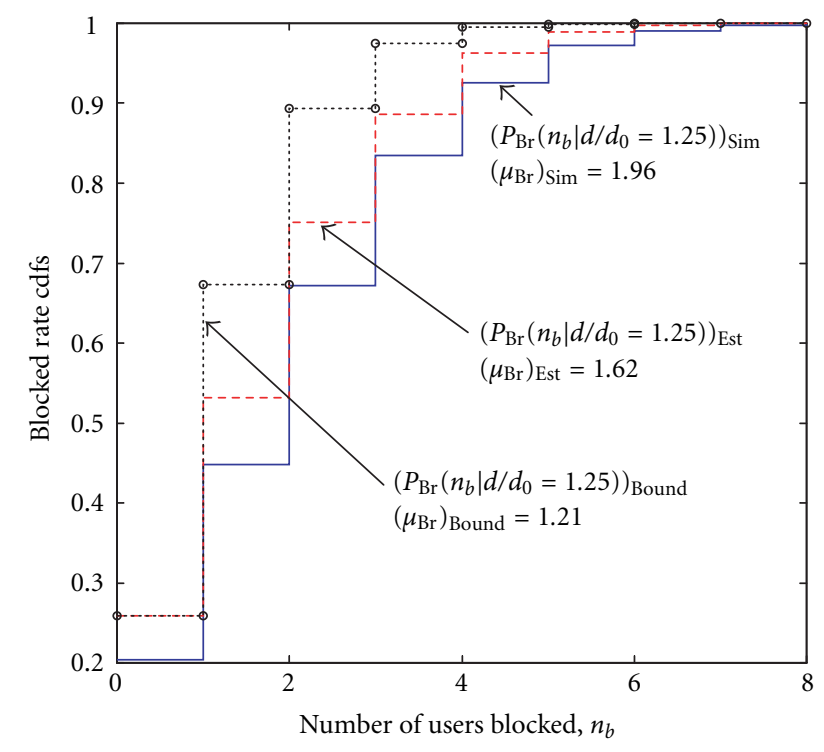

FIGURE 4: Blocking rate cdf comparison between the analytical upper bound, analytical estimate, and the cdf estimate based on the simulation model. Results are for a 1D network topology with $b=10, m=5, G_{c}=0.8, n=40$, and $d / d_{0}=1.25$.

TABLE 1: Comparison of expected blocking rates based on $b=10$ and $m=5$ for 1D model.

\begin{tabular}{|c|c|c|c|c|}
\hline \multicolumn{5}{|c|}{ Expected blocking rate } \\
\hline & \multicolumn{2}{|c|}{$G_{c}=0.4 \Rightarrow n=20$} & \multicolumn{2}{|c|}{$G_{c}=0.8 \Rightarrow n=50$} \\
\hline & $d / d_{0}=1.25$ & $d / d_{0}=2.0$ & $d / d_{0}=1.25$ & $d / d_{0}=2.0$ \\
\hline$\left(\mu_{\mathrm{Br}}\right)_{\text {Bound }}$ & 0.025 & $3.4 \times 10^{-12}$ & 1.2 & $7.0 \times 10^{-6}$ \\
\hline$\left(\mu_{\mathrm{Br}}\right)_{\mathrm{Est}}$ & 0.025 & $6.2 \times 10^{-7}$ & 1.6 & 0.64 \\
\hline$\left(\mu_{\mathrm{Br}}\right)_{\mathrm{Sim}}$ & 0.042 & $7.9 \times 10^{-4}$ & 2.0 & 0.25 \\
\hline
\end{tabular}

results obtained by the simulation were consistent with results obtained by both the bound and estimate.

\section{RESULTS}

Simulation results are presented in this section based on the Monte Carlo approach outlined in Section 3.1. In Figure 5, graphs for five pdfs, $p_{\mathrm{Br}}\left(n_{b} \mid d / d_{0}\right)$, are given. The pdfs are based on a $5 \times 5$ hexagonal grid of cells where, for each trial, $25 \mathrm{MSs}(25 \mathrm{BS} \times 1 \mathrm{MS} / \mathrm{BS})$ were uniformly distributed over the network coverage region. Then, based on the value of $d / d_{0}$ and the resulting MS to BS assignment, the number of blocked MSs, $n_{b}$, was recorded. Similar experiments were performed to obtain estimates for $p_{\mathrm{Br}}\left(n_{b} \mid d / d_{0}\right)$ over the desired parameter ranges. The pdf estimates were then used to determine the mean, $\mu_{\mathrm{Br}}\left(n_{b} / n \mid d / d_{0}\right)$, and standard deviation, $\sigma_{\mathrm{Br}}\left(n_{b} / n \mid d / d_{0}\right)$, of the blocking rate versus the offered traffic per channel, $G_{c}$. Figure 6 shows the results for the statistical analysis performed on the estimated pdfs with $m \in\{1,5,10,20\}$ and $d / d_{0} \in\{1,1.5,2.0\}$ obtained for

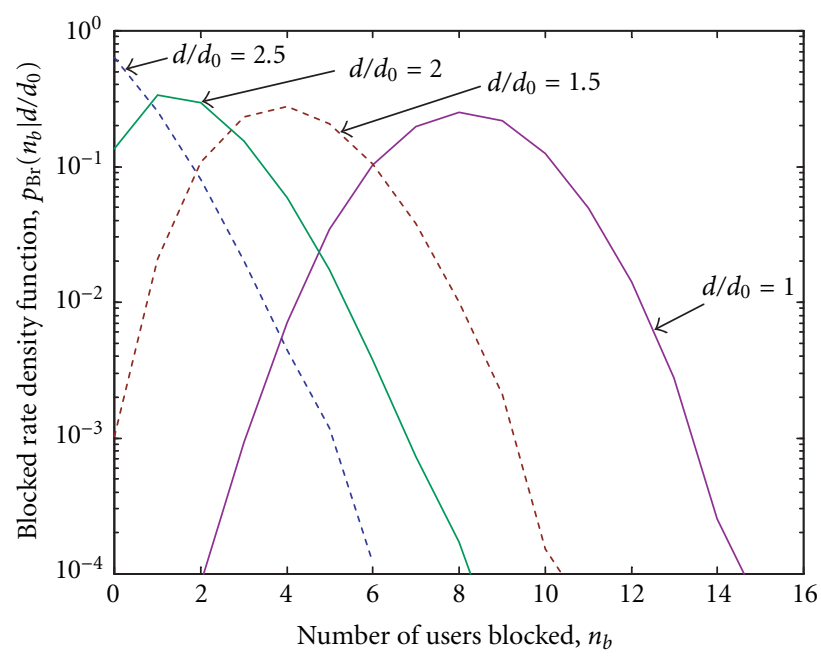

FIGURE 5: Estimate for the conditional blocking rate density function based on $b=25, m=1, G_{c}=1, n=25$, and Traffic Model I.

Traffic Model I. Note that $\mu_{\mathrm{Br}}\left(n_{b} \mid d / d_{0}\right)<10^{-3}$ for $[m \in$ $\left.\{5,10\}, d / d_{0}=2.0\right]$ and that $\left[m=20, d / d_{0} \in\{1.5,2.0\}\right]$ over the range of $G_{c}$ evaluated.

Figure 7 shows the results for the statistical analysis performed on the estimated pdfs obtained for Traffic Model II: $\sigma_{\operatorname{Tr}}=3 \mathrm{~dB}$ in graphs (a) and (b) and $\sigma_{\mathrm{Tr}}=4 \mathrm{~dB}$ in graphs (c) and (d). Several observations can be made concerning the impact increasing the coverage range has on the grade of service (GoS), that is, the blocking rate probability. First, comparing $\mu_{\mathrm{Br}}\left(n_{b} / n \mid d / d_{0}=1.0\right)$ with $\mu_{\mathrm{Br}}\left(n_{b} / n \mid d / d_{0}=2.0\right)$ for $\sigma_{\mathrm{Tr}}=3 \mathrm{~dB}$ (Figure 7a), an order of magnitude improvement in GoS is obtained. By doubling the coverage range, all channels in the network are used to satisfy the offered traffic, $G_{c}=1.0$, on average. To achieve the same expected level of GoS with the standard cellular coverage, the offered traffic decreases to $G_{c} \approx 0.4$. Examining $\sigma_{\mathrm{Br}}$ for the same pdfs (Figure $7 \mathrm{~b}$ ), we can see that the slope of the $\sigma_{\mathrm{Br}}\left(n_{b} / n \mid d / d_{0}=\right.$ 2.0) graph dramatically falls off as $G_{c}$ decreases; whereas, the slope of $\sigma_{\mathrm{Br}}\left(n_{b} / n \mid d / d_{0}=1.0\right)$ is nearly constant, with a value exceeding $2 \%$ blocking. Therefore, if the blocking rate design criteria is based on a constraint to improve robustness, that is, $\mu+\beta \sigma, \beta>0$, then the impact on $G_{c}$ will be significantly less when range extension is employed.

Another interesting observation, based on Figure 7, is the effect of trunking. Doubling the number of channels per BS ( 5 to 10 or 10 to 20 ) does not significantly impact the relative performance until $G_{c}$ drops below a certain level. Since the traffic load is not uniform over the network coverage region, MSs have a higher probability of being grouped within a region, that is, a hot spot. Adding additional channel capacity uniformly over the entire network does not solve the problem. On the other hand, with $d / d_{0}$ sufficiently large, channel capacity is available to handle the offered traffic, while reducing the number of channels at each BS.

To illustrate the impact on capacity, the desired GoS was set at a $2 \%$ blocking rate, with a $95 \%$ probability, $\operatorname{Pr}\left(n_{b} / n \leq\right.$ $0.02)=0.95$. The estimated pdfs were then evaluated to 


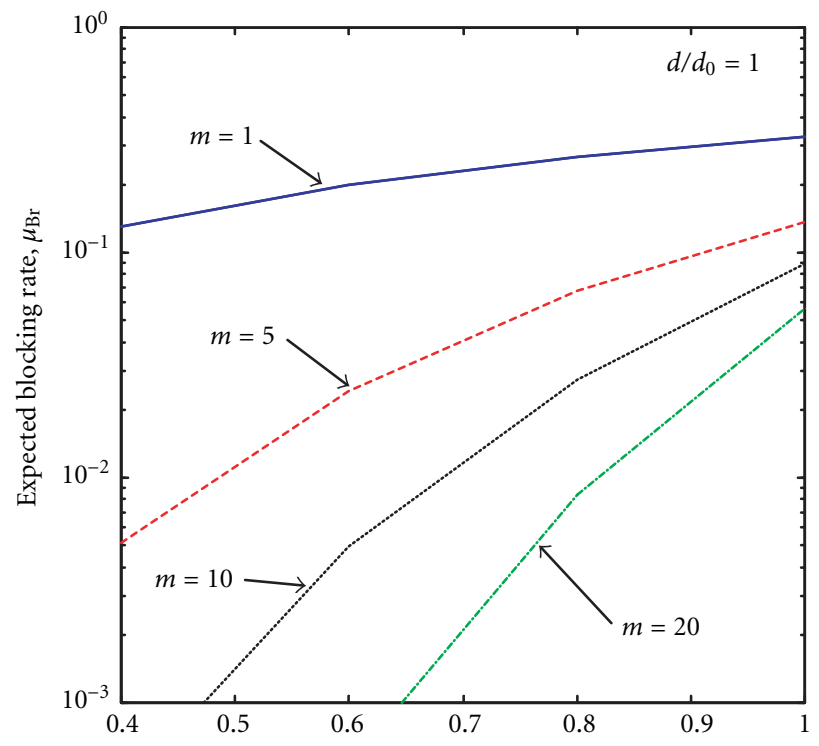

(a) Offered traffic per channel, $G_{c}$.

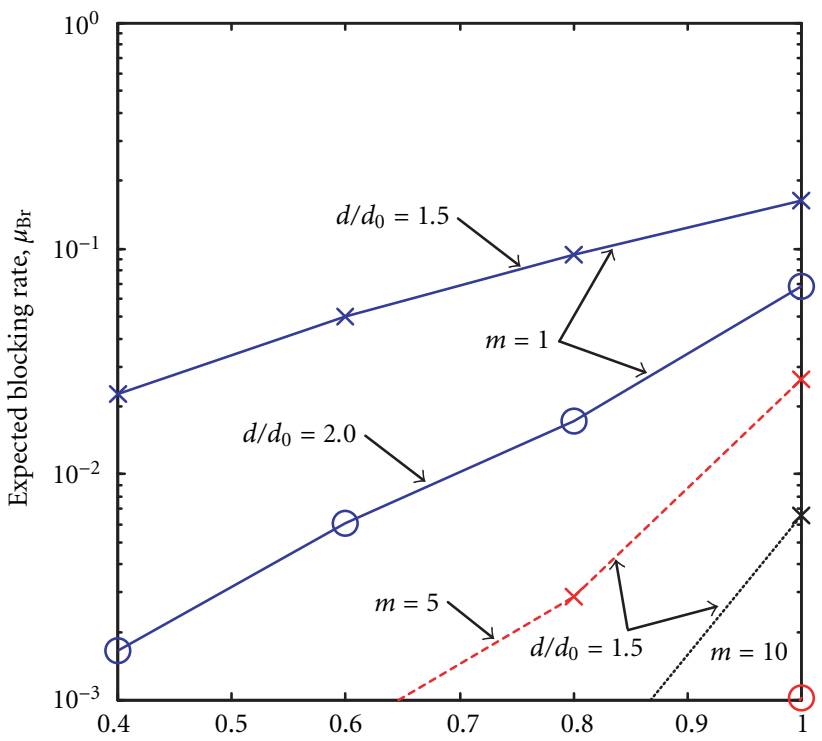

(c) Offered traffic per channel, $G_{c}$.

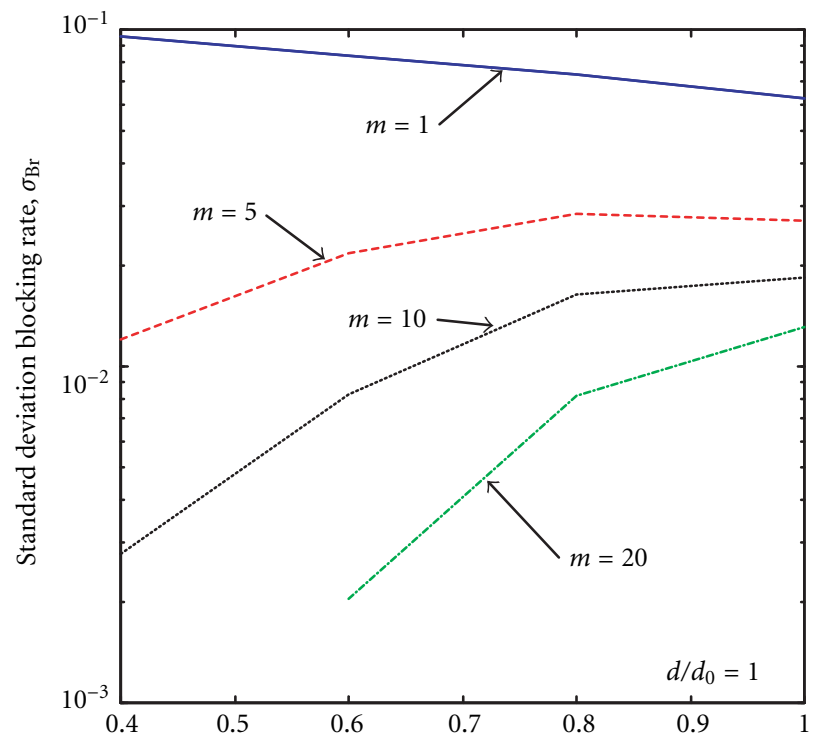

(b) Offered traffic per channel, $G_{c}$.

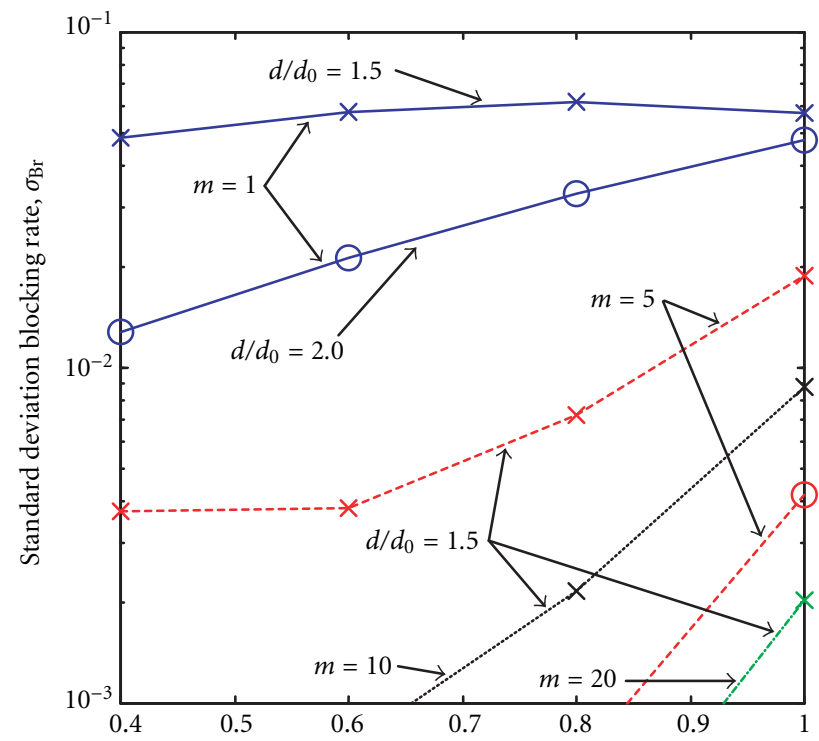

(d) Offered traffic per channel, $G_{c}$.

FiguRE 6: Statistical analysis for blocking rate simulations with uniform distributed cell traffic load, Traffic Model I.

determine the probability for each experiment conducted. The results are shown in Table 2, based on the percentage of the maximum network capacity utilized, given that the specified GoS was satisfied. The maximum network capacity was the total number of channels available in the network (third column of the table). The results for both traffic models are shown in the table. The same trends as noted in the previous paragraphs can also be observed. That is, a significant improvement occurs in the capacity as $d / d_{0}$ increases and no significant change in the relative efficiency occurs when the number of trunks increases $(m>1$ and log-normal traffic distribution). Again, focusing on $\sigma_{\mathrm{Tr}}=3 \mathrm{~dB}$, for $m=5,10$, or 20 with a $50 \%$ extension in range, the capacity can be more than doubled. Extending the range by $250 \%$ results in a $100 \%$ utilization of the network resources, while still satisfying the specified GoS. Hence, a 4-fold capacity increase is achieved.

As evident from the results in Figure 6 and Table 2, for the uniform traffic distribution, increasing the number of 
TABle 2: Percentage of the maximum capacity utilized by the network based on a GoS blocking probability of $2 \%$.

\begin{tabular}{|c|c|c|c|c|c|c|}
\hline \multirow{2}{*}{$\sigma_{\operatorname{Tr}}$} & \multirow{2}{*}{$m$} & \multirow{2}{*}{ Max. \# channels } & \multicolumn{4}{|c|}{$\%$ Maximum capacity } \\
\hline & & & $d / d_{0}=1$ & $d / d_{0}=1.5$ & $d / d_{0}=2$ & $d / d_{0}=2.5$ \\
\hline \multirow{4}{*}{ Uniform } & 1 & 25 & $<10$ & 31 & 50 & 81 \\
\hline & 5 & 125 & 40 & 81 & 100 & 100 \\
\hline & 10 & 250 & 60 & 95 & 100 & 100 \\
\hline & 20 & 500 & 73 & 100 & 100 & 100 \\
\hline \multirow{4}{*}{$3 \mathrm{~dB}$} & 1 & 25 & 17 & 29 & 63 & 85 \\
\hline & 5 & 125 & 25 & 56 & 81 & 100 \\
\hline & 10 & 250 & 25 & 63 & 81 & 100 \\
\hline & 20 & 500 & 26 & 64 & 81 & 100 \\
\hline \multirow{4}{*}{$4 \mathrm{~dB}$} & 1 & 25 & 6 & 24 & 46 & 72 \\
\hline & 5 & 125 & 19 & 42 & 58 & 82 \\
\hline & 10 & 250 & 20 & 45 & 61 & 84 \\
\hline & 20 & 500 & 20 & 44 & 60 & 83 \\
\hline
\end{tabular}

channels at each BS provides a relative improvement in performance, for all $d / d_{0}$. For this traffic model, given that the BSs have a sufficient number of channels, load balancing provides only a marginal benefit. An economic benefit is possible, since fewer channels could be deployed at each BS to achieve the desired GoS. This benefit would need to be balanced against the complexity of implementing the algorithm to achieve load balancing. From the results in Figure 7 and Table 2, for the nonuniform traffic distribution, a threshold is reached beyond which adding additional channels provides little or no relative improvement in performance. Under this condition, load balancing using adaptive antenna arrays to extend coverage range could provide a significant enhancement.

\section{CONCLUSIONS}

In this paper, we have introduced an alternative method for optimizing the adaptive antenna arrays at the BSs in a cellular system. The method was based on adapting the array weights to maximize $\eta_{C} \times \eta_{T}$. An upper bound was formulated for evaluating the impact on spectral efficiency. To assess the bound, the effectiveness of using an adaptive antenna array to maximize the trunking efficiency was evaluated. Monte Carlo simulations were used to evaluate the trunking efficiency and the corresponding impact on capacity. For a linear grid cellular system, both theoretical analysis and Monte Carlo simulations were developed. The Monte Carlo simulations results were consistent with the theoretical results. Based on the simulation results for a 2D network with a uniform traffic distribution, the performance improvement gained by load balancing is dependent on the number of available channels at the BS. For the nonuniform traffic model, the results were significantly different. Over a $200 \%$ improvement in capacity was achieved when the adaptive antenna array was used to extend the coverage range by $50 \%$ and a $400 \%$ capacity improvement for a range extension of $250 \%$. These results were not dependent on the relative number of available channels at the BS. Based on (3) and (4), the full advantage of load balancing is achieved when excess spatial channels from one cell are utilized to support traffic activity in neighboring cells, such that the overall traffic carried by the network is increased.

A portion of the research presented in this paper appeared in [34].

\section{APPENDICES}

\section{A. STATISTICAL ANALYSIS USING 1D NETWORK}

The following 1D network model, as illustrated in Figure 2, was used for the statistical analysis. A linear grid cellular network topology of $b$ cell sites was considered with cell- 1 adjacent to cell-b. The BS coverage range was $d_{0}$ with an omnidirectional antenna and $d$ with an adaptive antenna array. Each cell could support up to $m$ traffic channels. At a given instant in time, $n$ users required a traffic channel,

$$
n=b m G_{c}
$$

where $G_{c}$ is the offered traffic per channel in the network. The approach used in the derivation was based on an instantaneous analysis of the network activity. Therefore, the dynamics of the user call activity were modeled as a lumped parameter, $G_{c}$. The $n$ users were assumed to be uniformly and independently distributed within the coverage region of the $b$ cell sites.

\section{A.1. Statistical analysis using 1D network topology and $d / d_{0}=1$}

The blocking rate was evaluated by letting the RV $n_{b}$ represent the number of users not serviced within the network at a given instant in time. Then, using the model defined in the previous paragraph, a closed form solution was derived for 


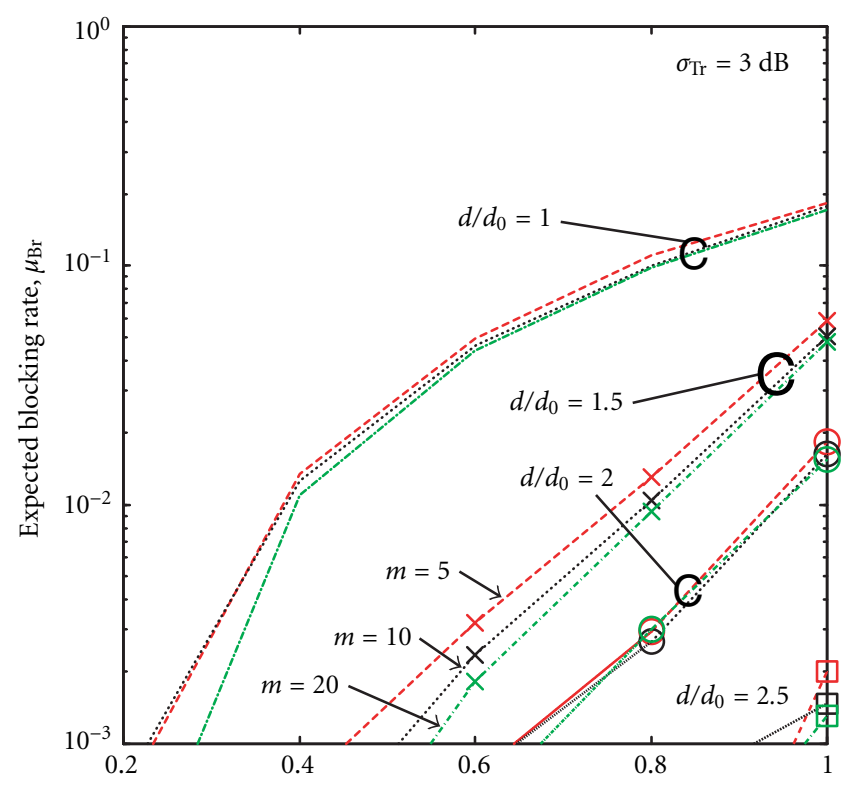

(a) Offered traffic per channel, $G_{c}$.

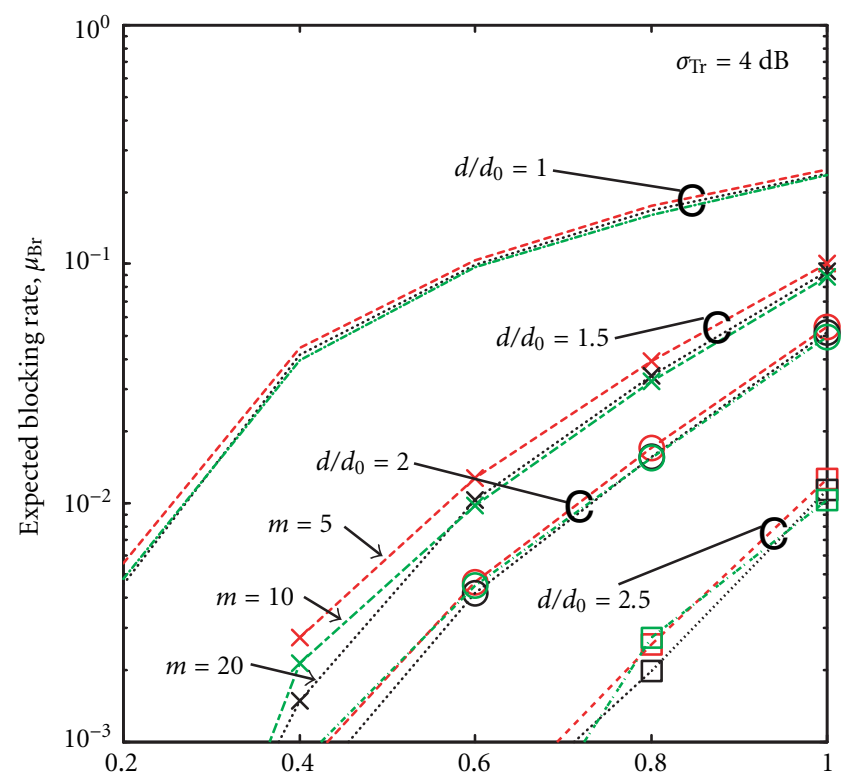

(c) Offered traffic per channel, $G_{c}$.

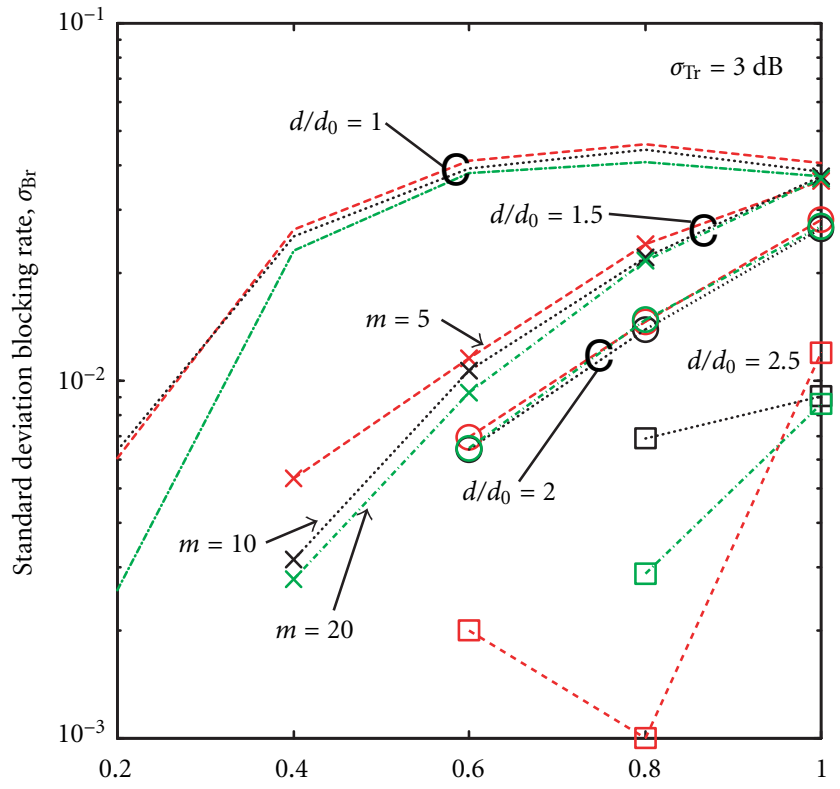

(b) Offered traffic per channel, $G_{c}$.

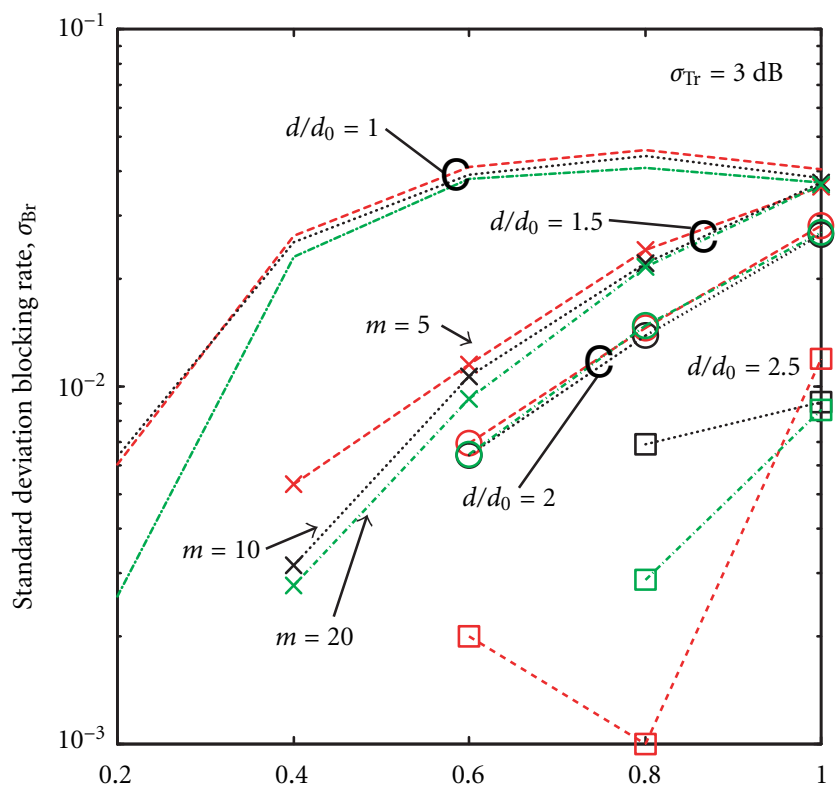

(d) Offered traffic per channel, $G_{c}$.

Figure 7: Statistical analysis for blocking rate simulations with log-normal distributed cell traffic load: (a) and (b) log-normal traffic load standard deviation, $\sigma_{\mathrm{Tr}}=3 \mathrm{~dB}$; (c) and (d) $\sigma_{\mathrm{Tr}}=3 \mathrm{~dB}$.

the cdf for $n_{b}$ when $d / d_{0}=1.0$,

$$
P_{\mathrm{Br}}\left(n_{b} \mid d / d_{0}=1.0\right)=\sum_{j=0}^{n-1} \operatorname{Pr}_{\mathrm{Br}}\left(n_{b}=j \mid d / d_{0}=1.0\right) u\left(n_{b}-j\right)
$$

where $u(\cdot)$ is the unit step function; $\operatorname{Pr}_{\mathrm{Br}}\left(n_{b}=j \mid d / d_{0}=\right.$ $1.0)$ is the probability that $j$ users are blocked due to excess traffic channels required in one or more cell sites when an omnidirectional antenna is employed at the BS. With a linear network topology, uniformly distributed users and equal area cells, evaluating $\operatorname{Pr}_{\mathrm{Br}}\left(n_{b}=j \mid d / d_{0}=1.0\right)$ required 
enumerating the permutations and combinations for $n_{b}=j$ based on $n$ users and $b$ cell sites as derived below.

Let $c_{k}$ be an ordered $b$-tuple, representing one of the possible combinations of $n$ users in $b$ cell sites where

$$
c_{k}=\left(c_{k_{1}}, c_{k_{2}}, \ldots, c_{k_{b}}\right), \quad 1 \leq k \leq s_{\mathcal{c}}, 0 \leq c_{k_{i}} \leq n, 1 \leq i \leq b .
$$

In addition, the following constraints are required on the elements of $c_{k}: c_{k_{i}} \geq c_{k_{j}}, \forall i<j$ and $\sum_{i=1}^{b} c_{k_{i}}=n$. The total number of ordered combinations is $s_{c}$. For each combination $c_{k}$, the number of blocked users, $n_{b_{k}}$, is given by

$$
n_{b_{k}}=\sum_{i=1}^{b} \max \left(0, c_{k_{i}}-m\right), \quad 0 \leq n_{b_{k}}<n .
$$

Next, let $r_{j}$ be the set of combinations such that the number of blocked users in each combination is equal to $j$, that is,

$$
r_{j}=\left\{c_{k} \mid n_{b_{k}}=j, 0 \leq j<n-1,1 \leq k \leq s_{c}\right\} .
$$

Note that $r_{i} \cap r_{j}=\phi$ and $\bigcup_{i=0}^{n-1} r_{i}=\bigcup_{k=1}^{s_{c}} c_{k}$. Then, the blocking rate probability can be expressed as

$$
\operatorname{Pr}_{\mathrm{Br}}\left(n_{b}=j \mid d / d_{0}=1.0\right)=\sum_{c_{k} \in r_{j}} x_{k} y_{k},
$$

where $x_{k}$ is the number of permutations for combination $c_{k}$ and $y_{k}$ is the corresponding probability for $c_{k}$.

For each combination $c_{k}, x_{k}$ is the number of unique ways the elements of $c_{k}$ may be reordered amongst the $b$ cell sites, given by

$$
x_{k}=\prod_{i=1}^{n}\left(\begin{array}{c}
b-\sum_{j=1}^{i=1} w_{k_{j}} \\
w_{k_{i}}
\end{array}\right),
$$

where $w_{k_{i}}$ represents the number of occurrences for a specific number of users, $i$, in $c_{k}$. This can be expressed as

$$
w_{k_{j}}=\left|\left\{c_{k_{j}} \mid c_{k_{j}} \in c_{k}, c_{k_{j}}=i, 1 \leq j \leq b\right\}\right|, \quad 1 \leq i \leq n,
$$

where $|\cdot|$ is the cardinality of a set.

The probability that the combination $c_{k}$ occurs is

$$
y_{k}=\prod_{i=1}^{b}\left(\begin{array}{c}
n-\sum_{j=1}^{i=1} c_{k_{j}} \\
c_{k_{i}}
\end{array}\right) q_{i}^{c_{k_{i}}}
$$

where $q_{i}$ is the a priori probability that a user is within the coverage range of cell site $i$. Since it is assumed that each cell has equal coverage area and that the $n$ users are uniformly and independently distributed, each cell site is equiprobable, $q=q_{1}=\cdots=q_{b}=1 / b$. Therefore,

$$
y_{k}=b^{-n} \prod_{i=1}^{b}\left(\begin{array}{c}
n-\sum_{j=1}^{i=1} c_{k_{j}} \\
c_{k_{i}}
\end{array}\right) .
$$

Thus, substituting the results of (A.5), (A.7), and (A.10) into (A.6), a closed form solution for $\operatorname{Pr}_{\mathrm{Br}}\left(n_{b}=j \mid d / d_{0}=1.0\right.$ ) is obtained. To illustrate: for $b=4, m=1$, and $G_{c}=1 \Rightarrow n=$ 4 , the

$$
\begin{aligned}
\operatorname{Pr}_{\mathrm{Br}} & \left(n_{b}=2 \mid d / d_{0}=1.0\right) \\
= & x_{2} y_{2}+x_{3} y_{3} \\
= & {\left[\left(\begin{array}{l}
4 \\
1
\end{array}\right)\left(\begin{array}{l}
3 \\
1
\end{array}\right)\right]\left[4^{-4}\left(\begin{array}{l}
4 \\
3
\end{array}\right)\left(\begin{array}{l}
1 \\
1
\end{array}\right)\right] } \\
& +\left[\left(\begin{array}{l}
4 \\
2
\end{array}\right)\right]\left[4^{-4}\left(\begin{array}{l}
4 \\
2
\end{array}\right)\left(\begin{array}{l}
2 \\
2
\end{array}\right)\right],
\end{aligned}
$$

since $r_{j=2}=\left\{c_{2} c_{3}\right\}=\{(3,1,0,0)(2,2,0,0)\}$. Note that the combinations involving 0 have been excluded from (A.11). In this fashion, (A.6) can be evaluated over the range for $n_{b}$, $0 \leq n_{b}<n$, to obtain (A.2), $P_{\mathrm{Br}}\left(n_{b} \mid d / d_{0}=1.0\right.$ ).

\section{A.2. Statistical bound using 1D network topology and $d / d_{0} \geq 1$}

In this section, an upper bound for the blocking rate cdf for $d / d_{0}>1$ is derived based on the 1D network model:

$$
\left(P_{\mathrm{Br}}\left(n_{b} \mid d / d_{0}=(1.0+\alpha)\right)\right)_{\text {Bound }} \geq P_{\mathrm{Br}}\left(n_{b} \mid d / d_{0}=(1.0+\alpha)\right),
$$

where $0.0<\alpha<1.0$. Using the same notation as used in deriving (A.2), $\left(P_{\mathrm{Br}}\left(n_{b} \mid d / d_{0}=(1.0+\alpha)\right)\right)_{\text {Bound }}$ is obtained by determining a lower bound on the number of blocked users, $\bar{n}_{b_{k}} \leq n_{b_{k}}$, for each combination $c_{k}$ of $n$ users in $b$ cells. Therefore, for $c_{k}$ there are $c_{k_{i}}$ users within cell $i$ distributed within three regions. Referring to Figure 2, these regions are designated L, C, and R. Since the users are uniformly distributed in the coverage region, the $c_{k_{i}}$ users are uniformly distributed within cell $i$. Let $\eta_{l}=\left(\eta_{l_{1}}, \eta_{l_{2}}, \eta_{l_{3}}\right)$ be a 3-tuple, representing one of the possible combinations of the $c_{k_{i}}$ users, where $\eta_{l_{1}}$, $\eta_{l_{2}}$, and $\eta_{l_{3}}$ are the number of users in region- $\mathrm{L}$, region-C, and region-R, respectively. Note that the index $k_{i}$ has been dropped from $\eta_{l}$ in order to simplify the notation. All possible 3-tuples are considered, which satisfy $\sum_{z=1}^{3} \eta_{l_{z}}=c_{k_{i}}$ and $\eta_{l_{z}} \geq 0, \forall z$. The total number of combinations is $s_{\eta}$. For each combination $\eta_{l}$, the number of blocked users, $\beta_{l}$, is given by

$$
\begin{aligned}
\beta_{l}= & \max \left(0, \eta_{l_{2}}-M_{C}\right) \\
& +\max \left(0, \eta_{l_{1}}-\left(M_{L}+\max \left(0, M_{C}-\eta_{l_{2}}\right)\right)\right) \\
& +\max \left(0, \eta_{l_{3}}-\left(M_{R}+\max \left(0, \max \left(0, M_{C}-\eta_{l_{2}}\right)\right.\right.\right. \\
& \left.\left.\left.-\max \left(0, \eta_{l_{1}}-M_{L}\right)\right)\right)\right),
\end{aligned}
$$

where the adjacent BSs provide $M_{L}$ and $M_{R}$ channels to regions $L$ and $R$, respectively, and the cell $i$ BS provides $M_{C}$ channels that can be used to provide service anywhere in the cell. The first term in (A.13) expresses the number of blocked users in region-C, which can only be serviced by the cell's BS. The second term expresses the number of blocked users in region- $\mathrm{L}$, where the channels provided by the left adjacent BS are used in preference to those available from the cell's BS. The last term, in a similar fashion, determines the blocked users in region- $R$. 
In general, $M_{L}, M_{c}$, and $M_{R}$ are dependent on the specific permutation of the ordered $b$-tuple $c_{k}$ and therein lies the difficulty in determining the general solution to $P_{\mathrm{Br}}\left(n_{b} \mid d / d_{0}=\right.$ $(1.0+\alpha))$. In order to determine $\left(P_{\mathrm{Br}}\left(n_{b} \mid d / d_{0}=(1.0+\right.\right.$ $\alpha)))_{\text {Bound }}$, a lower bound on the blocking rate for $\eta_{l}, \bar{\beta}_{l} \leq \beta_{l}$, is required. Letting $M_{L}=M_{C}=M_{R}=m$ in (A.13) gives $\bar{\beta}_{l} \leq \beta_{l}$. Then a lower bound on the number of users blocked in cell $i$, given $c_{k_{i}}$ users, is

$$
\bar{n}_{b_{k}, i}=\left.\sum_{l=1}^{s_{\eta}}\left(\operatorname{Pr}\left(\eta_{l}\right) \bar{\beta}_{l}\right)\right|_{c_{k_{i}}} \leq n_{b_{k}, i}
$$

where

$$
\begin{aligned}
\operatorname{Pr}\left(\eta_{l}\right)= & (\alpha / 2)^{\eta_{l_{1}}}(1-\alpha / 2)^{\eta_{l_{2}}}(\alpha / 2)^{\eta_{l_{3}}} \\
& \times\left(\begin{array}{c}
c_{k_{i}} \\
\eta_{l_{1}}
\end{array}\right)\left(\begin{array}{c}
c_{k_{i}}-\eta_{l_{1}} \\
\eta_{l_{2}}
\end{array}\right)\left(\begin{array}{c}
c_{k_{i}}-\eta_{l_{1}}-\eta_{l_{2}} \\
\eta_{l_{3}}
\end{array}\right) .
\end{aligned}
$$

Using (A.14), a lower bound on the number of users blocked for $c_{k}$ is

$$
\bar{n}_{b_{k}}=\sum_{i=1}^{b} \bar{n}_{b_{k}, i} \leq n_{b_{k}}
$$

Using (A.16) in conjunction with (A.2), (A.3), (A.5), (A.6), (A.7), (A.8), (A.9), and (A.10) provides that $\left(p_{\mathrm{Br}}\left(n_{b} \mid d / d_{0}=\right.\right.$ $(1.0+\alpha))_{\text {Bound }}$.

The tightness of the blocking probability bound is dependent on the offered traffic, $G_{c}$. For low values of $G_{c}$, using $M_{L}=M_{C}=M_{R}=m$ is a reasonable assumption. For $G_{c}=1$, the bound is poor since the total number of channels available per cell is $3 m$ and therefore will significantly underestimate the number of users blocked.

A better approximation for the blocking probability can be obtained. Instead of assuming that $M_{L}=M_{R}=m$, the number of channels available from adjacent BSs is conditioned on the expected activity in each cell, that is, $n / b$. Using the expected activity, the unallocated channels in each cell can be estimated by

$$
\hat{M}=\max \left(0, m-\left\lceil\frac{n}{b}\right\rceil\right) .
$$

An estimate for the blocking probability, $\left(P_{\mathrm{Br}}\left(n_{b} \mid d / d_{0}=\right.\right.$ $(1.0+\alpha)))_{\text {Est }}$, can be obtained by using $M_{L}=M_{R}=\hat{M}$, and $M_{C}=m$ in (A.13).

\section{B. MS TO BS ASSIGNMENT ALGORITHM}

Heuristic used to determine the MS to BS assignment in order to approximate $\min \left(n_{b}\right)$.

Define:

$M_{j} \equiv$ unassigned mobile $j$,

$B_{i} \equiv$ base station $i, i=1, \ldots, b$,

$\left(M_{j}^{*}, B_{i}^{*}\right) \equiv$ Assignment of $M_{j}$ to $B_{i}$ to $\min \left(n_{b}\right)$,

$C\left(B_{i}\right) \equiv$ Set of unassigned traffic channels at $B_{i}$,

$D\left(M_{j}, B_{i}\right) \equiv$ Distance between $M_{j}$ and $B_{i}$,
$N_{C}\left(M_{j}\right) \equiv\left|\cup C\left(B_{i}\right)\right| D\left(M_{j}, B_{i}\right) \leq d \mid$, that is, number of traffic channels to which $M_{j}$ could be assigned, and $|\cdot|$ is the cardinality of a set, $R_{B}\left(B_{i}\right) \equiv$ $\left|C\left(B_{i}\right)\right| /\left|\left\{M_{j} \mid D\left(M_{j}, B_{i}\right) \leq d, \forall j\right\}\right|$, that is, For $B_{i}$, the ratio of the number of unassigned channels at $B_{i}$ by the number of unassigned mobiles within distance $d$ of the base station.

Repeat the following until all mobiles $M_{j}, j=1, \ldots, n$ are either assigned or blocked:

(1) $G=\left\{M_{j} \mid \operatorname{argmin}_{M_{j}}\left(N_{C}\left(M_{j}\right)\right)\right\}$, determine the set of unassigned mobiles with the minimum number of possible unassigned traffic channels,

(2) $\left(M_{j}^{*}, B_{i}^{*}\right)=\operatorname{argmax}_{B_{i}, G}\left(R_{B}\left(B_{i}\right) \mid M_{j} \in G, D\left(M_{j}, B_{i}\right) \leq d\right)$, make the base station to mobile assignment based on the unassigned mobiles from step (1) and the assignment that maximizes $R_{B}\left(B_{i}\right)$,

(3) Update set of unassigned channels and unassigned mobiles based on assignment from step (2) and remove blocked traffic, that is, $N_{C}\left(M_{j}\right)=0 \Rightarrow M_{j}$ is blocked.

\section{REFERENCES}

[1] G. V. Tsoulos, G. E. Athanasiadou, J. P. McGeehan, and M. Beach, "Adaptive antennas for UMTS microcellular operational environments," in Proc. IEEE 48th Vehicular Technology Conference, vol. 2, pp. 895-898, Ottawa, Canada, 1998.

[2] P. Petrus, "Exploiting a new dimension-space," in $M P R G$ Propagator-Virginia Tech, vol. 10, pp. 2-3, 1999.

[3] G. Stüber, Principles of Mobile Communication, Kluwer Academic, Boston, Mass, USA, May 1996.

[4] L. Anderson, "A smart solution-the latest antenna technology provides cost-effective capacity gains," in Wireless Review, pp. 32-38, 1999.

[5] J. Winters, "Optimum combining in digital mobile radio with cochannel interference," IEEE Journal on Selected Areas in Communications, vol. 2, no. 4, pp. 528-539, 1984.

[6] J. Winters, "Optimum combining for indoor radio systems with multiple users," IEEE Trans. Communications, vol. 35, no. 11 , pp. 1222-1230, 1987.

[7] J. Winters, "Signal acquisition and tracking with adaptive arrays in the digital mobile radio system IS-54 with flat fading," IEEE Transactions on Vehicular Technology, vol. 42, no. 4, pp. 377-384, 1993.

[8] J. Winters, J. Salz, and R. D. Gitlin, "The impact of antenna diversity on the capacity of wireless communication systems," IEEE Trans. Communications, vol. 42, no. 4, pp. 1740-1751, 1994.

[9] J. Winters, "Adaptive antennas for wireless systems-short course notes," in Proc. IEEE Vehicular Technology Conference, Washington, D.C., USA, 1997.

[10] J. Winters, "Smart antennas for wireless systems," IEEE Personal Communications, vol. 5, no. 1, pp. 23-27, 1998.

[11] S. Swales, M. Beach, D. Edwards, and J. McGeehan, "The performance enhancement of multibeam adaptive base-station antennas for cellular land mobile radio systems," IEEE Transactions on Vehicular Technology, vol. 39, no. 1, pp. 56-66, 1990.

[12] M. Beach, P. Guémas, and A. Nix, "Capacity and service extension for wireless networks using adaptive antennas," Electronics Letters, vol. 20, no. 22, pp. 1813-1814, 1994.

[13] G. Tsoulos, M. Beach, and S. Swales, "Sensitivity analysis of capacity enhancement with adaptive multibeam antennas for 
DCS 1800," Electronics Letters, vol. 32, no. 19, pp. 1745-1747, 1996.

[14] G. V. Tsoulos, M. A. Beach, and S. C. Swales, "On the sensitivity of the capacity enhancement of a TDMA system with adaptive multibeam antennas," in Proc. IEEE 47th Vehicular Technology Conference, vol. 1, pp. 165-169, Phoenix, Ariz, USA, 1997.

[15] K. Ivanov, C. Lüders, and U. Rehfueb, "Estimating and comparing the upper bounds on the capacity gain from different smart antenna concepts in GSM mobile radio networks," in Proc. IEEE 48th Vehicular Technology Conference, pp. 499-504, Ottawa, Canada, 1998.

[16] P. Petrus, I. Howitt, and H. H. Reed, "Evaluation of outage probability due to cochannel interference in fading for an AMPS system with an ideal beamformer," in Proc. 8th International Conference on Wireless Communications, vol. 1, Calgary, Canada, July 1996.

[17] Y. Hawwar, "Cochannel interference analysis with a beamformer for TDMA cellular system," M.S. thesis, EECS, University of Wisconsin-Milwaukee, Milwaukee, Wis, USA, 1997.

[18] I. Howitt and Y. Hawwar, "Evaluation of outage probability due to cochannel interference in fading for a TDMA system with a beamformer," in Proc. IEEE 48th Vehicular Technology Conference, Ottawa, Canada, 1998.

[19] P. Petrus, R. Ertel, and J. Reed, "Capacity enhancement using adaptive arrays in an AMPS system," IEEE Transactions on Vehicular Technology, vol. 47, no. 3, pp. 717-727, 1998.

[20] J. Fuhl, A. Kuchar, and E. Bonek, "Capacity increase in cellular PCS by smart antennas," in Proc. IEEE 47th Vehicular Technology Conference, vol. 3, pp. 1962-1966, Phoenix, Ariz, USA, May 1997.

[21] J. Liberti and T. S. Rappaport, "Analytical results for capacity improvements in CDMA," IEEE Transactions on Vehicular Technology, vol. 43, no. 3, pp. 680-690, 1994.

[22] J. Liberti, Analysis of CDMA Cellular Radio Systems Employing Adaptive Antennas, Ph.D. thesis, EE, Virginia Polytechnic Institute and State University, Blacksburg, 1995.

[23] W. Ye and A. Haimovich, "Outage probability of cellular CDMA systems with space diversity, Rayleigh fading and power control error," IEEE Communications Letters, vol. 2, no. 8, pp. 220-222, 1998.

[24] G. Tsoulos, M. Beach, and S. Swales, "DS-CDMA capacity enhancement with adaptive antennas," Electronics Letters, vol. 31, no. 16, pp. 1319-1320, 1995.

[25] C. K. Kim and Y. S. Cho, "Capacity improvement of a MCCDMA cellular system with antenna arrays in a fading channel," in Proc. IEEE 48th Vehicular Technology Conference, vol. 3, pp. 2032-2036, Ottawa, Canada, 1998.

[26] A. M. Earnshaw and S. D. Blostein, "Efficient evaluation of adaptive digital beamforming for multi-service provision in a cellular CDMA system," in Proc. IEEE 48th Vehicular Technology Conference, vol. 3, pp. 1665-1669, Ottawa, Canada, 1998.

[27] J. Winters and M. Gans, "The range increase of adaptive versus phased arrays in mobile radio systems," IEEE Transactions on Vehicular Technology, vol. 48, no. 2, pp. 353-362, 1989.

[28] I. Howitt, "Match filter bound analysis used to evaluate range increase provided by an adaptive antenna array," in Proc. IEEE Vehicular Technology Conference, Boston, Mass, USA, Fall 2000.

[29] European Telecommunications Standardization Institute, "UMTS selection procedures for the choice of radio transmission technologies of the UMTS-UMTS 30.03 version 3.2.0," ETSI TR 101112 v3.2.0 (1998-04), 1998.

[30] E. Nikula, A. Toskala, E. Dahlman, L. Girard, and A. Klein, "Frames multiple access for UMTS and IMT-2000," IEEE Personal Communications, vol. 5, no. 2, pp. 16-24, 1998.
[31] J. Zander, "Radio resource management in future wireless networks- requirements and limitations," IEEE Communications Magazine, vol. 5, no. 8, pp. 30-35, 1997.

[32] A. Baier, U.-C. Fiebig, W. Granzow, W. Koch, P. Teder, and J. Thielecke, "Design study for a CDMA-based 3rd generation mobile radio system," IEEE Journal on Selected Areas in Communications, vol. 12, no. 9, pp. 733-742, 1994.

[33] U. Gotzner, A. Gamst, and R. Rathgeber, "Spatial traffic distribution in cellular networks," in Proc. IEEE 48th Vehicular Technology Conference, vol. 3, pp. 1994-1998, Ottawa, Canada, 1998.

[34] I. Howitt and F. H. Awad, "Capacity enhancement based on using adaptive antenna arrays optimized for trunking efficiency," in Proc. IEEE Vehicular Technology Conference, Boston, Mass, USA, 2000.

Ivan Howitt received the BSEE and MS degrees in electrical engineering from Georgia Institute of Technology in 1982 and 1990, respectively, and the Ph.D. degree in electrical engineering from University of California, Davis in 1995. From 1982 to 1990, he was a Research Engineer with Georgia Tech Research Institute. In 1995 to 1996, he was a Visiting Assistant Professor at Virginia Tech and a research associate at the Mobile and Portable Radio Research Group (MPRG). In 1996, he joined the Department of Electrical Engineering \& Computer Science at the University of Wisconsin Milwaukee (UWM). At UWM, he is the Director of the Wireless Communications \& Signal Processing Laboratory. His research interests include coexistence issues facing UL band wireless services and methods for mitigating interference; in addition, to his interest in optimization strategies for using adaptive antenna arrays in both UL band and cellular wireless services.

Fahed Awad received his B.S. degree in electrical and computer engineering from the University of Science and Technology, Irbid, Jordan in 1990 and his M.S. degree in electrical engineering from the University of Wisconsin Milwaukee in 1994. From 1992 to 1996, he worked as a teaching and research assistant at the university of Wisconsin Milwaukee. From 1996 to 2001, he worked as a switching and network design

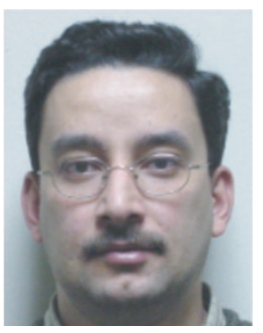
engineer in the cellular service provider industry. Since 2001, he has been working as a research engineer in the Advanced Technology Laboratory at Rockwell Automation. He is currently a Ph.D. candidate in the Department of electrical Engineering and Computer Science at the University of Wisconsin Milwaukee. His research interests are in the area of wireless communication networks. 